\title{
A Study of Generalized Thermoelastic Interaction in an Infinite Fibre-Reinforced Anisotropic Plate Containing a Circular Hole
}

\author{
IBrAhim A. ABBAS ${ }^{a, b, *}$ AND ABO-EL-NOUR N. ABD-ALla ${ }^{a, b}$ \\ ${ }^{a}$ Department of Mathematics, Faculty of Science, Sohag University, Sohag, Egypt \\ ${ }^{b}$ Department of Mathematics, Faculty of Science, Jazan University, Jazan, Saudi Arabia
}

(Received May 7, 2010; in final form November 23, 2010)

\begin{abstract}
In the context of Lord and Shulman theory, the generalized thermoelastic theory with thermal relaxation is used to investigate the thermoelastic interaction in an infinite fibre-reinforced anisotropic plate containing a circular hole. Thermoelastic interactions are caused by a uniform step at temperature applied to the boundary of the hole which is stress-free. The problem is solved numerically using a finite element method and the numerical solutions of the non-dimensional governing partial differential equations of the problem are shown graphically. The effects of the presence and absence reinforcement on temperature, stress and displacement are studied.
\end{abstract}

PACS: 46.25.Hf, 81.05.Ni, 02.70.Dh

\section{Introduction}

Materials such as resins reinforced by strong aligned fibres exhibit highly anisotropic elastic behaviour in a sense that their elastic moduli for extension in the fibre direction are frequently of the order of 50 or more times greater than their elastic moduli in transverse extension or in shear. The mechanical behaviour of many fibre-reinforced composite materials is adequately modelled by the theory of linear elasticity for transversely isotropic materials, with the preferred direction coinciding with the fibre direction. In such composites the fibres are usually arranged in parallel straight lines. However, other configurations are used. An example is that of circumferential reinforcement, for which the fibres are arranged in concentric circles, giving strength and stiffness in the tangential (or hoop) direction.

The theory of strongly anisotropic materials has been extensively discussed in the literature; Belfield et al. [1] studied the stress in elastic plates reinforced by fibres lying in concentric circles.

Sengupta and Nath [2] discussed the problem of surface waves in fibre-reinforced anisotropic elastic media. Singh [3] showed that, for wave propagation in fibre-reinforced anisotropic media, this decoupling cannot be achieved by the introduction of displacement potentials.

Hashin and Rosen [4] gave the elastic moduli for fibre-reinforced materials. In the classical dynamical coupled theory of thermoelasticity, thermal and mechanical waves propagate with an infinite velocity, which is not physically admissible.

The theory of couple thermoelasticity was extended by Lord and Shulman (LS) [5] and Green and Lindsay [6]

* corresponding author; e-mail: ibrabbas7@yahoo.com by including the thermal relaxation time in constitutive relations. The theory was extended for anisotropic body by Dhaliwal and Sherief [7].

Singh [8] studied the wave propagation in thermally conducting linear fibre-reinforced composite materials with one relaxation time.

Verma [9] discussed the problem of magnetoelastic shear waves in self-reinforced bodies. Chattopadhyay and Choudhury [10] investigated the propagation, reflection and transmission of magnetoelastic shear waves in self-reinforced media.

Chattopadhyay and Choudhury [11] studied the propagation of magnetoelastic shear waves in an infinite self-reinforced plate. Chattopadhyay and Michel [12] studied a model for spherical second harmonic $(\mathrm{SH})$-wave propagation in self-reinforced linearly elastic media. The exact solution of the governing equations of the generalized thermoelasticity theory for a coupled and nonlinear/linear system exists only for very special and simple initial and boundary problems. A numerical solution technique is used to calculate the solution of general problems. For this reason the finite element method is chosen.

The finite element method is a powerful technique originally developed for numerical solution of complex problems in structural mechanics, and it remains the method of choice for complex systems. A further benefit of this method is that it allows physical effects to be visualized and quantified regardless of experimental limitations. On the other hand, the finite element method in different generalized thermoelastic problems has been applied by many authors [13-17].

In this paper, a general finite element model is proposed to analyze transient phenomena in thermoelastic model in the context of the theory of generalized thermoelasticity with one relaxation time. We consider thermoelastic problem involving such circumferentially 
reinforced plates. The composite material is then locally transversely isotropic, with the direction of the axis of transverse isotropy not constant, but everywhere directed along the tangents to circles in which the fibres lie. The problem is solved numerically using a finite element method. Moreover, the numerical solutions of the non-dimensional governing partial differential equations of the problem are shown graphically. Results carried out in this paper can be used to design various fibre-reinforced anisotropic thermoelastic elements under thermal load to meet special engineering requirements.

\section{Problem formulation: governing equations}

In the context of the LS-theory, the field equations for linear equations governing thermoelastic interactions in a fiber-reinforced linearly thermoelastic anisotropic medium whose preferred direction is that of a unit vector $\boldsymbol{a}$, in the absence of body forces and heat sources, are as follows [8]:

$$
\begin{aligned}
& \tau_{i j, j}=\rho \ddot{u}_{i}, \quad i, j=1,2,3 \\
& K_{i j} T_{, i j}=\rho c_{\mathrm{e}}\left(\dot{T}+\tau_{0} \ddot{T}\right)+T_{0} \beta_{i j}\left(\dot{u}_{i, j}+\tau_{0} \ddot{u}_{i, j}\right) \\
& \quad i, j=1,2,3 \\
& \quad \tau_{i j}=\lambda e_{k k} \delta_{i j}+2 \mu_{T} e_{i j}+\alpha\left(a_{k} a_{m} e_{k m} \delta_{i j}+a_{i} a_{j} e_{k k}\right) \\
& \quad+2\left(\mu_{L}-\mu_{T}\right)\left(a_{i} a_{k} e_{k j}+a_{j} a_{k} e_{k i}\right) \\
& \quad+\beta a_{k} a_{m} e_{k m} a_{i} a_{j}-\beta_{i j}\left(T-T_{0}\right) \delta_{i j} \\
& \quad i, j, k, m=1,2,3
\end{aligned}
$$

where $\rho$ is the mass density, $u_{i}$ is the displacement vector components, $e_{i j}$ is the strain tensor, $\tau_{i j}$ is the stress tensor, $T$ is temperature change of a material particle, $T_{0}$ is the reference uniform temperature of the body, $\beta_{i j}$ is the thermal elastic coupling tensor, $c_{\mathrm{e}}$ is the specific heat at constant strain, $K_{i j}$ is the thermal conductivity, $t_{0}$ is the relaxation time, $\lambda, \mu_{T}$ are elastic parameters, $\alpha, \beta,\left(\mu_{L}-\mu_{T}\right)$ are reinforced anisotropic elastic parameters and the components of the vector $\boldsymbol{a}$ are $\left(a_{1}, a_{2}, a_{3}\right)$, where $a_{1}^{2}+a_{2}^{2}+a_{3}^{2}=1$. The comma notation is used for spatial derivatives and superimposed dot represents time differentiation. For circumferential reinforcement, it is normal to employ a system of cylindrical polar coordinates $(r, \theta, z)$ and henceforth all components are referred to these coordinates. In this system, for cylindrical symmetric interactions, the displacement vector possesses only the radial component $u=u(r, t)$, where $r$ is the radial distance measured from the origin (point of symmetry), and the stress tensor is determined by the radial stress $\tau_{r r}$ and the circumferential stress (hoop stress) $\tau_{\theta \theta}$. For circumferential reinforcement, the vector $\boldsymbol{a}$ is everywhere directed in the tangential (i.e. $\theta$ ) direction, thus in cylindrical polar coordinates $\boldsymbol{a}$ has components $(0,1,0)$. In this case, Eqs. (1)-(3) yield the following governing equations for $u$ and $T$ :

$$
\begin{aligned}
& \frac{\partial \tau_{r r}}{\partial r}+\frac{1}{r}\left(\tau_{r r}-\tau_{\theta \theta}\right)=\rho \frac{\partial^{2} u}{\partial t^{2}}, \\
& K_{11} \frac{\partial^{2} T}{\partial r^{2}}+K_{22} \frac{1}{r} \frac{\partial T}{\partial r}=\left(\frac{\partial}{\partial t}+t_{0} \frac{\partial^{2}}{\partial t^{2}}\right) \\
& \quad \times\left(\rho c_{\mathrm{e}} T+T_{0} \beta_{11} \frac{\partial u}{\partial r}+T_{0} \beta_{22} \frac{u}{r}\right), \\
& \tau_{r r}=\left(\lambda+2 \mu_{T}\right) \frac{\partial u}{\partial r}+(\lambda+\alpha) \frac{u}{r}-\beta_{11}\left(T-T_{0}\right), \\
& \tau_{\theta \theta}=(\lambda+\alpha) \frac{\partial u}{\partial r}+\left(\lambda+2 \alpha+4 \mu_{L}-2 \mu_{T}+\beta\right) \frac{u}{r} \\
& \quad-\beta_{22}\left(T-T_{0}\right),
\end{aligned}
$$

with $\beta_{11}=2\left(\lambda+\mu_{T}\right) \alpha_{11}+(2 \lambda+\alpha) \alpha_{22}, \beta_{22}=2(\lambda+$ $\alpha) \alpha_{11}+\left(\lambda+2 \alpha+4 \mu_{L}-2 \mu_{T}+\beta\right) \alpha_{22}$, where $\alpha_{11}, \alpha_{22}$ are coefficients of linear thermal expansion. For convenience, the following non-dimensional variables are used:

$$
\begin{aligned}
& \left(r^{\prime}, u^{\prime}\right)=c_{1} \chi(r, u), \quad\left(t^{\prime}, t_{0}^{\prime}\right)=c_{1}^{2} \chi\left(t, t_{0}\right), \chi=\frac{\rho c_{\mathrm{e}}}{K_{1}}, \\
& \left(\tau_{r r}^{\prime}, \tau_{\theta \theta}^{\prime}\right)=\frac{1}{A}\left(\tau_{r r}, \tau_{\theta \theta}\right), T^{\prime}=\frac{T-T_{0}}{T_{0}}, c_{1}=\sqrt{\frac{A}{\rho}}, \\
& A=\lambda+2 \alpha+4 \mu_{L}-2 \mu_{T}+\beta .
\end{aligned}
$$

In terms of the non-dimensional quantities defined in Eqs. (8), the above governing equations reduce to (dropping the dash for convenience):

$$
\begin{aligned}
& \frac{\partial}{\partial r}\left(S_{1} \frac{\partial u}{\partial r}+S_{2} \frac{u}{r}-S_{3} T\right)+\left(S_{1}-S_{2}\right) \frac{1}{r} \frac{\partial u}{\partial r} \\
& \quad+\left(S_{2}-1\right) \frac{u}{r^{2}}-\left(S_{3}-S_{4}\right) \frac{T}{r}=\frac{\partial^{2} u}{\partial t^{2}}, \\
& \frac{\partial^{2} T}{\partial r^{2}}+\varepsilon_{1} \frac{1}{r} \frac{\partial T}{\partial r}=\left(\frac{\partial}{\partial t}+t_{0} \frac{\partial^{2}}{\partial t^{2}}\right)\left(T+\varepsilon_{2} \frac{\partial u}{\partial r}+\varepsilon_{3} \frac{u}{r}\right), \\
& \tau_{r r}=S_{1} \frac{\partial u}{\partial r}+S_{2} \frac{u}{r}-S_{3} T, \\
& \tau_{\theta \theta}=S_{2} \frac{\partial u}{\partial r}+\frac{u}{r}-S_{4} T, \\
& \left(S_{1}, S_{2}, S_{3}, S_{4}\right)=\frac{1}{A}\left(\lambda+2 \mu_{T}, \lambda+\alpha, T_{0} \beta_{11}, T_{0} \beta_{22}\right), \\
& \left(\varepsilon_{1}, \varepsilon_{2}, \varepsilon_{3}\right)=\left(\frac{K_{22}}{K_{11}}, \frac{\beta_{11}}{\rho c_{\mathrm{e}}}, \frac{\beta_{22}}{\rho c_{\mathrm{e}}}\right) .
\end{aligned}
$$

where

\section{Boundary conditions}

The surface of the hole i.e. $r=1$ is assumed to be stress free and is subjected to a uniform step in temperature effect so that the boundary conditions are taken as

$$
\tau_{r r}(1, t)=0, \quad T(1, t)=H(t),
$$

where $H(t)$ denotes the Heaviside unit step function.

Initially the medium is at rest and undisturbed and the initial conditions are

$$
u(r, 0)=\frac{\partial u(r, 0)}{\partial t}=0, \quad T(r, 0)=\frac{\partial T(r, 0)}{\partial t}=0 .
$$




\section{Solution of the problem}

The finite element equations of a generalized thermoelasticity problem can be readily obtained by the following standard procedure. In the finite element method, the displacement component $u$ and temperature $T$ are related to the corresponding nodal values by

$$
u=\sum_{i=1}^{m} N_{i} u_{i}(t), \quad T=\sum_{i=1}^{m} N_{i} T_{i}(t),
$$

where $m$ denotes the number of nodes per element, and $N$ is the shape functions. In the framework of standard Galerkin procedure, the weighting functions and the shape functions coincide. Thus,

$$
\delta u=\sum_{i=1}^{m} N_{i} \delta u_{i}, \quad \delta T=\sum_{i=1}^{m} N_{i} \delta T_{i} .
$$

With Eqs. (15) and (16), $u^{\prime}=u_{, i}$ and $T^{\prime}=T_{, i}$ can be expressed as

$$
\begin{array}{ll}
u^{\prime}=\sum_{i=1}^{m} N_{i}^{\prime} u_{i}(t), & T^{\prime}=\sum_{i=1}^{m} N_{i}^{\prime} T_{i}(t), \\
\delta u^{\prime}=\sum_{i=1}^{m} N_{i}^{\prime} \delta u_{i}, & \delta T^{\prime}=\sum_{i=1}^{m} N_{i}^{\prime} \delta T_{i} .
\end{array}
$$

Thus, the finite element equations corresponding to Eqs. (9) and (10) can be obtained as

$$
\begin{gathered}
\sum_{e=1}^{m e}\left(\left[\begin{array}{cc}
M_{11}^{e} & 0 \\
M_{21}^{e} & M_{22}^{e}
\end{array}\right]\left\{\begin{array}{c}
\ddot{u}^{e} \\
\ddot{T}^{e}
\end{array}\right\}+\left[\begin{array}{cc}
0 & 0 \\
C_{21}^{e} & C_{22}^{e}
\end{array}\right]\left\{\begin{array}{c}
\dot{u}^{e} \\
\dot{T}^{e}
\end{array}\right\}\right. \\
\left.+\left[\begin{array}{cc}
K_{11}^{e} & K_{12}^{e} \\
0 & K_{22}^{e}
\end{array}\right]\left\{\begin{array}{c}
u^{e} \\
T^{e}
\end{array}\right\}=\left\{\begin{array}{l}
F_{1}^{e} \\
F_{2}^{e}
\end{array}\right\}\right),
\end{gathered}
$$

where $M^{e}$ is the total number of elements. The coefficients in Eq. (19) are given below

$$
\begin{aligned}
& M_{11}^{e}=\int[N]^{\mathrm{T}}[N] \mathrm{d} r, \quad M_{22}^{e}=\int t_{0}[N]^{\mathrm{T}}[N] \mathrm{d} r, \\
& C_{22}^{e}=\int[N]^{\mathrm{T}}[N] \mathrm{d} r, \\
& M_{21}^{e}=\int t_{0}[N]^{\mathrm{T}}\left(\varepsilon_{2}\left[N^{\prime}\right]+\frac{\varepsilon_{3}}{r}[N]\right) \mathrm{d} r, \\
& C_{21}^{e}=\int[N]^{\mathrm{T}}\left(\varepsilon_{2}\left[N^{\prime}\right]+\frac{\varepsilon_{3}}{r}[N]\right) \mathrm{d} r, \\
& K_{11}^{e}=\int\left[\left[N^{\prime}\right]^{\mathrm{T}}\left(S_{1}\left[N^{\prime}\right]+\frac{S_{2}}{r}[N]\right)+[N]^{\mathrm{T}}\right. \\
&\left.\times\left(\frac{S_{2}-S_{1}}{r}\left[N^{\prime}\right]+\frac{1-S_{2}}{r^{2}}[N]\right)\right] \mathrm{d} r, \\
& K_{12}^{e}=\int\left[-\left[N^{\prime}\right]^{\mathrm{T}} S_{3}[N]+\frac{S_{3}-S_{4}}{r}[N]^{\mathrm{T}}[N]\right] \mathrm{d} r, \\
& F_{1}^{e}=\left.[N]^{\mathrm{T}} \bar{\tau}\right|_{1} ^{r},
\end{aligned}
$$

$$
K_{22}^{e}=\int\left[\left[N^{\prime}\right]^{\mathrm{T}}[N]-\frac{\varepsilon_{1}}{r}[N]^{\mathrm{T}}[N]\right] \mathrm{d} r, F_{2}^{e}=\left.[N]^{\mathrm{T}} \bar{q}\right|_{1} ^{r} .
$$

Symbolically, the discretized equations of Eqs. (19) can be written as

$$
M \ddot{d}+C \dot{d}+K d=F^{\mathrm{ext}},
$$

where $M, C, K$ and $F^{\text {ext }}$ represent the mass, damping, stiffness matrices and external force vectors, respectively; $d=[u T]^{\mathrm{T}}, \bar{\tau}$ represent the component of the traction, and $\bar{q}$ represents heat flux. On the other hand, the time derivatives of the unknown variables have to be determined by the Newmark time integration method (see Wriggers [18]).

\section{Numerical example}

To study the effect of reinforcement on wave propagation, we use the following physical constants for generalized fibre-reinforced thermoelastic materials [8]: $\rho=2660 \mathrm{~kg} / \mathrm{m}^{3}, \lambda=5.65 \times 10^{10} \mathrm{~N} / \mathrm{m}^{2}, \mu_{T}=$ $2.46 \times 10^{10} \mathrm{~N} / \mathrm{m}^{2}, \mu_{L}=5.66 \times 10^{10} \mathrm{~N} / \mathrm{m}^{2}, \alpha=$ $-1.28 \times 10^{10} \mathrm{~N} / \mathrm{m}^{2}, \beta=220.90 \times 10^{10} \mathrm{~N} / \mathrm{m}^{2}, \alpha_{11}=$ $0.017 \times 10^{-4} \mathrm{deg}^{-1}, \alpha_{22}=0.015 \times 10^{-4} \mathrm{deg}^{-1}, c_{e}=$ $0.787 \times 10^{3} \mathrm{~J} \mathrm{~kg}^{-1} \mathrm{deg}^{-1}, T_{0}=293 \mathrm{~K}, K_{11}=$ $0.0921 \times 10^{3} \mathrm{~J} \mathrm{~m}^{-1} \mathrm{~s}^{-1} \mathrm{deg}^{-1}, K_{22}=0.0963 \times$ $10^{3} \mathrm{~J} \mathrm{~m}^{-1} \mathrm{~s}^{-1} \mathrm{deg}^{-1}$.

Before going to the analysis, the grid independence test has been conducted and the results are presented in Table. The grid size has been refined and consequently the values of different parameters as observed from Table get stabilized. Further refinement of mesh size over 8000 elements does not change the values considerably, which is therefore accepted as the grid size for computing purpose.

Grid independent test $(t=0.5, r=2.5)$.

TABLE

\begin{tabular}{c|c|c|c|c}
\hline \hline $\begin{array}{c}\text { Mesh } \\
\text { size }\end{array}$ & $T \times 10^{-2}$ & $u \times 10^{-7}$ & $\tau_{r r} \times 10^{-6}$ & $\tau_{\theta \theta} \times 10^{-5}$ \\
\hline 1000 & 7.478356 & 2.684009 & -3.144748 & -3.412876 \\
3000 & 7.478387 & 2.684094 & -3.144977 & -3.412899 \\
5000 & 7.478389 & 2.684110 & -3.145019 & -3.412902 \\
7000 & 7.478390 & 2.684110 & -3.145036 & -3.412903 \\
8000 & 7.478390 & 2.684088 & -3.141949 & -3.412903
\end{tabular}

Here all the variables/parameters are taken in non-dimensional forms. The results for temperature, displacement, radial stress and hoop stress have been carried out by taking $T_{1}=1, t_{0}=0.05$ and $t=0.5$. From the results, no significant difference in the value of temperature is noticed with reinforcement (WRE) and without reinforcement (NRE). The reinforcement has a great effect on the distribution of displacement and stresses.

Figure 1 shows that the temperature decreases as $r$ increases. The displacement is negative at $r=1$ where 


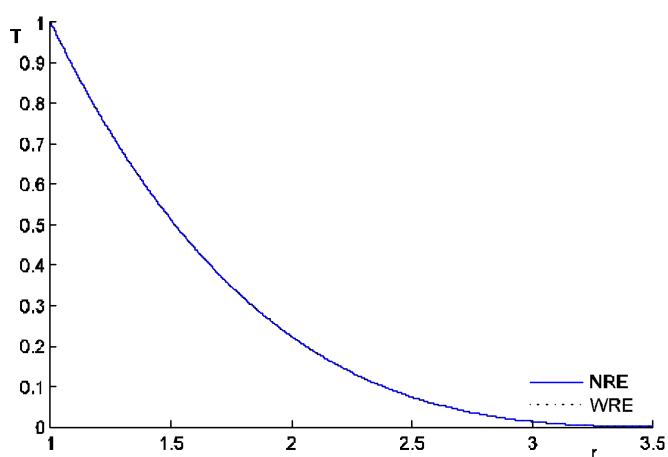

Fig. 1. The temperature distribution $T$ versus $r$ at $t=0.5$.

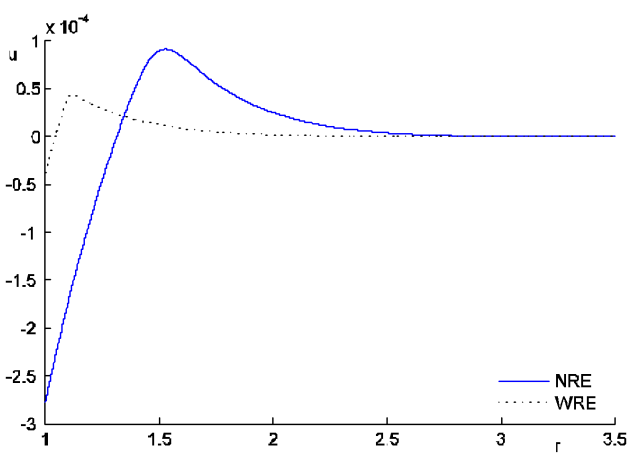

Fig. 2. The displacement distribution $u$ versus $r$ at $t=0.5$.

its magnitude is maximum as in Fig. 2. The displacement increases from the negative value to a positive value. In the positive values, the displacement has a peak value that depends on presence and absence reinforcement.

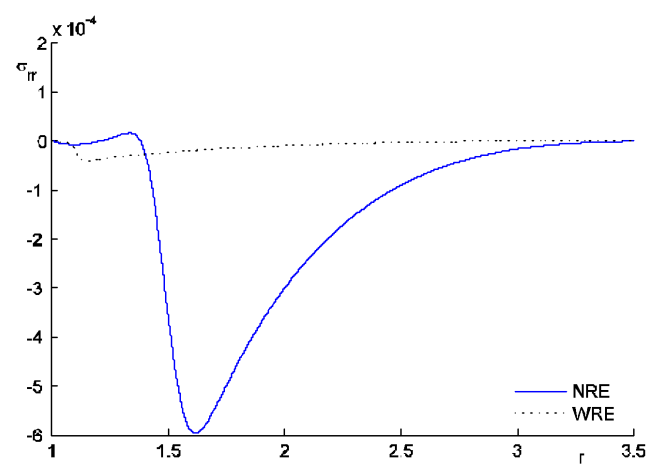

Fig. 3. The distribution of stress component $\sigma_{r r}$ versus $r$ at $t=0.5$.

Figures 3 and 4 depict that the variations of radial and hoop stress with respect to $r$ for presence and absence reinforcement in which we observed that the radial stress is zero at $r=1$ which satisfies the boundary conditions of the problem, the reinforcement has a great effect on the distribution of stresses.

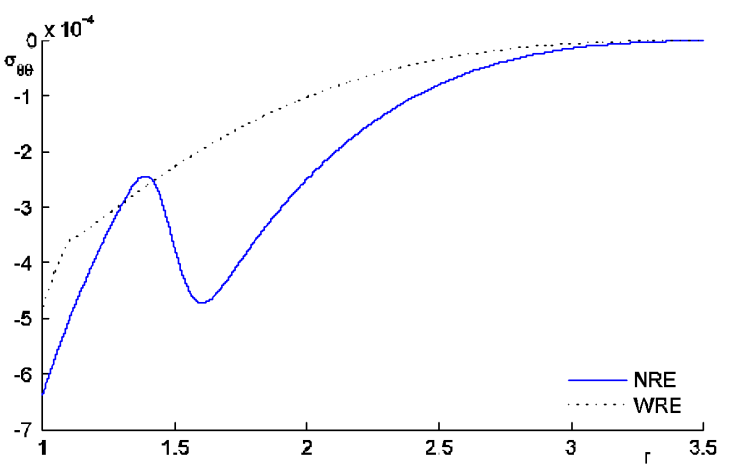

Fig. 4. The distribution of stress component $\sigma_{\theta \theta}$ versus $r$ at $t=0.5$.

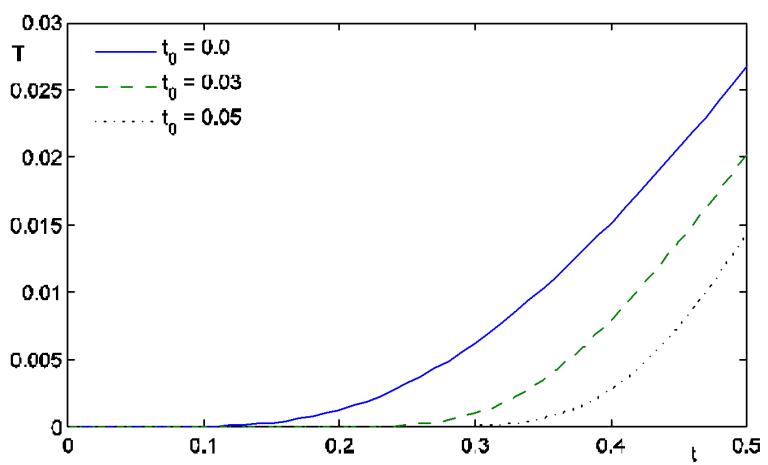

Fig. 5. The temperature distribution $T$ versus $t$ for different values of $t_{0}$ at $r=3$.

Figures 5-8 depict the variation of the displacement, the temperature, the radial stress and the hoop stress under two theories: classical dynamical coupled theory when $t_{0}=0$ and Lord and Shulman's theory when $t_{0}=$ $0.03,0.05$, with respect to time $t$ at $r=3$. It can be observed that the relaxation time has great effect on the distribution of field quantities.

\section{Conclusion}

In this paper we have investigated the generalized thermoelastic interaction in an infinite fibre-reinforced

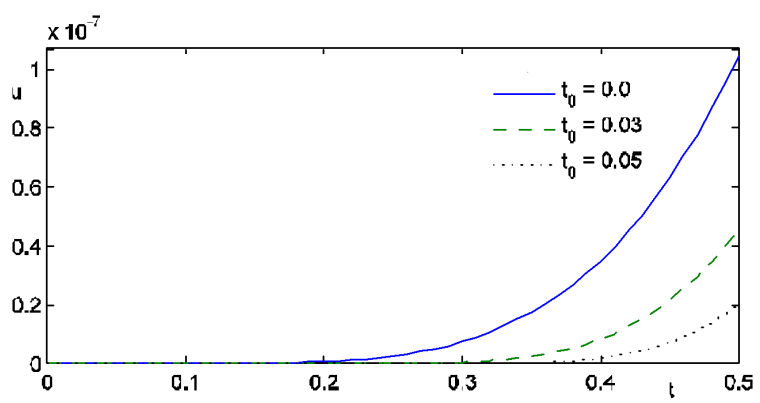

Fig. 6. The displacement distribution $u$ versus $t$ for different values of $t_{0}$ at $r=3$. 


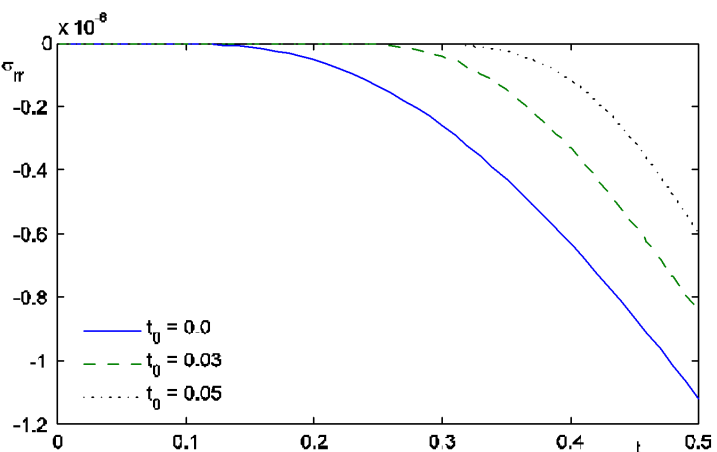

Fig. 7. The distribution of stress component $\sigma_{r r}$ versus $t$ for different values of $t_{0}$ at $r=3$.

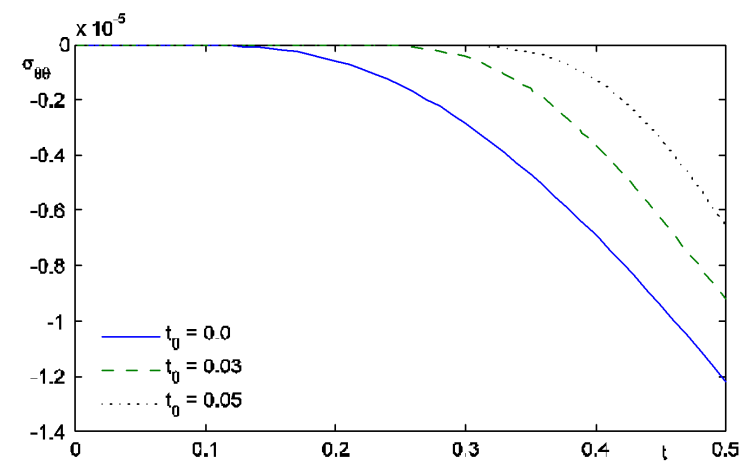

Fig. 8. The distribution of stress component $\sigma_{\theta \theta}$ versus $t$ for different values of $t_{0}$ at $r=3$.

anisotropic plate containing a circular hole with one relaxation time. The problem has been solved numerically using a finite element method (FEM). The differences of the field quantities predicted by the LS theory are remarkable in the presence and absence of reinforcement. The reinforcement has a great effect on the distribution of field quantities. The results which are carried out in this paper may be used to design various fibre-reinforced anisotropic thermoelastic elements under thermal load to meet special engineering requirements.

\section{References}

[1] A.J. Belfield, T.G. Rogers, A.J.M. Spencer, J. Mech. Phys. Solids 1, 25 (1983).

[2] P.R. Sengupta, S. Nath, Sãdhanã 26, 363 (2001).

[3] S.J. Singh, Sãdhanã 27, 1 (2002).

[4] Z. Hashin, W.B. Rosen, J. Appl. Mech. 31, 223 (1964).

[5] H. Lord, Y. Shulman, J. Mech. Phys. Solids 15, 299 (1967).

[6] A.E. Green, K.A. Lindsay, J. Elast. 2, 1 (1972).

[7] R.S. Dhaliwal, H.H. Sherief, Q. Appl. Math. 33, 1 (1980).

[8] B. Singh, Arch. Appl. Mech. 75, 513 (2006).

[9] P.D.S. Verma, Int. J. Eng. Sci. 24, 1067 (1986).

[10] A. Chattopadhyay, S. Choudhury, Int. J. Eng. Sci. 28, 485 (1990).

[11] A. Chattopadhyay, S. Choudhury, Int. J. Num. Anal. Methods Geomech. 19, 289 (1995).

[12] A. Chattopadhyay, V. Michel, Arch. Appl. Mech. 75, 113 (2006).

[13] X. Tian, Y. Shen, C. Chen, T. He, Int. J. Solids Struct. 43, 2050 (2006).

[14] I.A. Abbas, Forsch Ingenieurwes 71, 215 (2007).

[15] I.A. Abbas, A.N. Abd-alla, Arch. Appl. Mech. 78, 283 (2008).

[16] I.A. Abbas, M. Othman, Int. J. Indust. Math. 1, 121 (2009).

[17] H. Youssef, I.A. Abbas, Comp. Meth. Sci. Technol. 13, 95 (2007).

[18] P. Wriggers, Nonlinear Finite Element Methods, Springer, Berlin 2008. 